\title{
MicroRNA-217 inhibits the proliferation and invasion, and promotes apoptosis of non-small cell lung cancer cells by targeting sirtuin 1
}

\author{
GUANGSHUN LI and SHOUPING ZHONG
}

\author{
Department of Thoracic, Xi'an Central Hospital, Xi'an, Shaanxi 710003, P.R. China
}

Received December 4, 2019; Accepted October 22, 2020

DOI: $10.3892 / \mathrm{ol} .2021 .12647$

\begin{abstract}
Non-small cell lung cancer (NSCLC) is a common malignancy worldwide. MicroRNA (miR)-217 and sirtuin 1 (SIRT1) have been reported to play significant roles in different types of cancer, such as osteosarcoma and prostate cancer; however, the association between miR-217 and SIRT1 in the cell proliferation, apoptosis and invasion of NSCLC remain unknown. Thus, the present study aimed to investigate the roles of miR-217 and SIRT1 in NSCLC. The expression levels of miR-217 and SIRT1 were detected via reverse transcriptionquantitative (RT-q)PCR and western blot analyses. The effect of miR-217 on A549 and H1299 cell proliferation, apoptosis and invasion was assessed via the Cell Counting Kit-8, flow cytometry and Transwell assays, respectively. In addition, the association between SIRT1 and miR-217 was predicted using the TargetScan database, and verified via the dual-luciferase reporter assay, and RT-qPCR and western blot analyses. The results demonstrated that miR-217 expression was significantly downregulated, while SIRT1 expression was significantly upregulated in A549 and H1299 cells compared with the human bronchial epithelial cells. Furthermore, transfection with miR-217 mimic significantly inhibited A549 and H1299 cell proliferation and invasion, and induced A549 and H1299 cell apoptosis. The results of the dual-luciferase reporter assay and western blot analysis confirmed that SIRT1 is a target gene of miR-217. In addition, miR-217 inhibited the activation of AMP-activated protein kinase (AMPK) and mTOR signaling. Taken together, the results of the present study suggest that miR-217 inhibits A549 and H1299 cell proliferation and invasion, and induces A549 and H1299 cell apoptosis by targeting SIRT1 and inactivating the SIRT1-mediated AMPK/mTOR signaling pathway. Thus, miR-217 may be used as a potential therapeutic target for the treatment of patients with NSCLC.
\end{abstract}

Correspondence to: Dr Shouping Zhong, Department of Thoracic, Xi'an Central Hospital, 161 Xiwu Road, Xi'an, Shaanxi 710003, P.R. China

E-mail: zhongshoupingxazh@163.com

Key words: miR-217, non-small cell lung cancer, sirtuin 1

\section{Introduction}

Non-small cell lung cancer (NSCLC) is an aggressive malignancy worldwide $(1,2)$, with an incidence rate of $\sim 0.02 \%$ (3). Surgery in combination with chemotherapy and/or radiotherapy is the primary therapeutic regimen for patients with NSCLC in the clinic (4); however, the 5-year survival rate for these patients remains low at $\sim 15 \%$ (5), which is due to the aggressive nature of NSCLC (6). Thus, further investigations into factors which may be involved in the process of metastasis remain a priority to determine novel therapies for patients with NSCLC.

MicroRNAs (miRNAs/miRs) are a group of endogenous and non-coding RNAs, 22 nucleotides in length, which control the translation of target genes post-transcriptionally (7). During the initiation and development of different types of cancer, including lung cancer and gastric cancer, miRNAs have been reported to play crucial roles in downregulating oncogenes or tumor suppressors, thus regulating the cell proliferation, apoptosis and metastasis of cancer cells $(8,9)$. Notably, miR-217 has been identified to act as a tumor suppressor in lung cancer cells (10). In addition, miR-217 expression is downregulated by cigarette smoke extract (CSE) in human bronchial epithelial (HBE) cells (11).

As a $\mathrm{NAD}^{+}$-dependent histone deacetylase, sirtuin 1 (SIRT1) is universally expressed in the intracellular and extracellular matrix (12). Notably, SIRT1 expression is associated with a poor prognosis in patients with NSCLC (13). In addition, SIRT1 expression is upregulated in the brain tissues of patients with metastatic NSCLC, which subsequently promotes NSCLC cell migration (14). miR-217 has been demonstrated to decrease the development of osteosarcoma through SIRT1 (15). However, whether miR-217 inhibits the progression of NSCLC by targeting SIRT1 remains unknown. Taken together, the results of the present study demonstrated that miR-217 inhibited cell proliferation and invasion, and induced cell apoptosis of NSCLC cell lines by targeting SIRT1.

\section{Materials and methods}

Cell culture. HBE cells and the four NSCLC cell lines (H23, H292,H1299 and A549) were purchased from the American Type Culture Collection. All cells were maintained in RPMI-1640 medium supplemented with $10 \%$ fetal bovine serum (FBS) and $1 \%$ penicillin/streptomycin (all purchased from Invitrogen; Thermo Fisher Scientific, Inc.), at $37^{\circ} \mathrm{C}$ with $5 \% \mathrm{CO}_{2}$. 
Celltransfection.pcDNA3.1 orpcDNA3.1-SIRT1 reconstructed vectors (Guangzhou RiboBio Co., Ltd.) were transfected into H1299 and A549 cells using Lipofectamine ${ }^{\circledR} 2000$ reagent (Invitrogen; Thermo Fisher Scientific, Inc.), according to the manufacturer's protocol.

For the mimic transfections, cells were seeded into 6-well plates at a density of $1 \times 10^{5}$ cells/well and cultured in complete culture medium (Thermo Fisher Scientific, Inc.) overnight at $37^{\circ} \mathrm{C}$ with $5 \% \mathrm{CO}_{2}$. Following incubation, $50 \mathrm{nM}$ miR-217 mimic or miR-negative control (NC) mimic (Shanghai GenePharma Co., Ltd.) were transfected into H1299 and A549 cells using Lipofectamine ${ }^{\circledR} 2000$ reagent, according to the manufacturer's protocol. Following incubation for $24 \mathrm{~h}$ at $37^{\circ} \mathrm{C}$, cells were harvested for subsequent experimentation.

Cell Counting Kit-8 (CCK-8) assay. Cell proliferation was assessed via the CCK- 8 assay (Dojindo Molecular Technologies, Inc.), according to the manufacturer's protocol. Briefly, H1299 and A549 cells were seeded into a 96-well plate at a density of $5 \times 10^{3}$ cells/well and $10 \mu \mathrm{l}$ CCK- 8 solution was added to each well for 3, 0,24 and $48 \mathrm{~h}$ post-transfection. Cell proliferation was measured at a wavelength of $450 \mathrm{~nm}$, using a microplate reader (Bio-Rad Laboratories, Inc.).

Apoptosis analysis. $\mathrm{H} 1299$ and A549 cells $\left(1 \times 10^{6}\right.$ cells $\left./ \mathrm{ml}\right)$ were resuspended in $100 \mu \mathrm{l}$ binding buffer provided in the FITCAnnexin Vapoptosisdetectionkit(cat.no.556570;BDBiosciences), and subsequently treated with $5 \mu \mathrm{l}$ FITC-Annexin $\mathrm{V}$ or propidium iodide for $15 \mathrm{~min}$ at room temperature in the dark. The reaction was terminated following addition of $400 \mu \mathrm{l}$ binding buffer, and apoptotic cells were subsequently analyzed using a FACSCalibur cytometer (BD Biosciences) and CellQuest software (version 5.1; Becton, Dickinson and Company).

Cell invasion assay. Cell invasion was determined using Transwell chambers (8- $\mu \mathrm{m}$ pore; BD Biosciences). Briefly, $5 \times 10^{4}$ $\mathrm{H} 1299$ and A549 cells were plated in the Matrigel-coated (at $37^{\circ} \mathrm{C}$ for $5 \mathrm{~h}$ ) upper chambers, in serum-free RPMI-1640 medium. RPMI-1640 medium $(500 \mu \mathrm{l})$ supplemented with 10\% FBS was plated in the lower chambers. Following incubation for $24 \mathrm{~h}$ at $37^{\circ} \mathrm{C}$, the non-invasive cells were removed from the upper chambers using cotton swabs, while the invasive cells in the lower chambers were fixed with $100 \%$ methanol for $1 \mathrm{~min}$ at room temperature and stained with $0.1 \%$ crystal violet for $15 \mathrm{~min}$ at room temperature. Stained cells were counted in five randomly selected fields using a light microscope (magnification, x100).

Dual-luciferase reporter assay. The binding site between miR-217 and the 3'-untranslated region (UTR) of SIRT1 was predicted using the online TargetScan database (www.targetscan.org). The 3'-UTRs of wild-type (WT) and mutant (MT) SIRT1 were reconstructed into the $X b a \mathrm{I}$ site of the pGL3 vector (Promega Corporation). Subsequently, cells were seeded into a 24 -well plate at a density of $1 \times 10^{4}$ cells/well and co-transfected with $0.4 \mathrm{mg}$ pGL3-WT-SIRT1 or pGL3-MT-SIRT1 and/or $20 \mathrm{nM} \mathrm{miR-217}$ or miR-NC, using Lipofectamine ${ }^{\circledR} 2000$ reagent, according to the manufacturer's protocol. Following incubation for $48 \mathrm{~h}$ at $37^{\circ} \mathrm{C}$, cells were harvested and luciferase activities were detected using the Dual-Luciferase ${ }^{\circledR}$ Reporter Assay

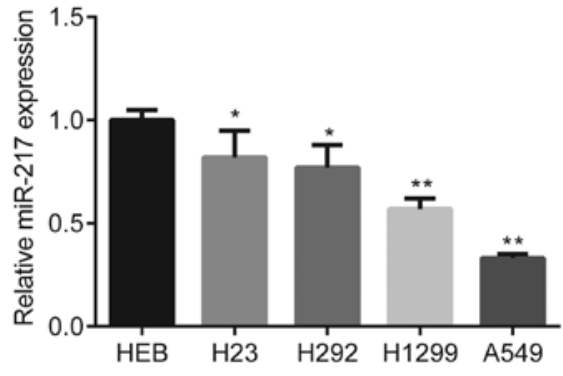

Figure 1. miR-217 expression in the NSCLC cell lines. miR-217 expression was downregulated in the NSCLC cell lines (H23, H292, H1299 and A549) compared with HBE cells. ${ }^{*} \mathrm{P}<0.05,{ }^{* *} \mathrm{P}<0.01$ vs. HEB. miR, microRNA; NSCLC, non-small cell lung cancer.

kit (cat. no. E1910; Promega Corporation). Firefly luciferase activity was normalized to Renilla luciferase activity.

Reverse transcription-quantitative (RT-q)PCR. Total RNA was extracted from HBE, H23, H292, H1299 and A549 cells using TRIzol ${ }^{\circledR}$ reagent (Invitrogen; Thermo Fisher Scientific, Inc.). Total RNA was reverse transcribed into cDNA using the cDNA Reverse Transcription kit (cat. no. 4368813, Applied Biosystems; Thermo Fisher Scientific, Inc.), according to the manufacturer's protocol. miR-217 expression was detected using the mirVana ${ }^{\mathrm{TM}}$ qRT-PCR miRNA Detection kit (cat. no. AM1558, Ambion; Thermo Fisher Scientific, Inc.), and normalized to the internal reference gene U6. PCNA, E-cadherin, vimentin and SIRT1 expression levels were detected using the SYBR Premix Ex Taq ${ }^{\mathrm{TM}}$ kit (cat. no. DRR041A, Takara Bio, Inc.), and normalized to the internal reference gene $\beta$-actin. qPCR was performed using the Applied Biosystems 7900 Fast Real-Time PCR system (Thermo Fisher Scientific, Inc.) The following thermocycling conditions were used for qPCR: Initial denaturation at $95^{\circ} \mathrm{C}$ for $5 \mathrm{~min}$; followed by 40 cycles of $95^{\circ} \mathrm{C}$ for $15 \mathrm{sec}, 60^{\circ} \mathrm{C}$ for $15 \mathrm{sec}$ and $72^{\circ} \mathrm{C}$ for $32 \mathrm{sec}$. The following primer sequences were used for qPCR: miR-217 forward, 5'-AGGTTAGTCAAG GACTAGCTCA-3, and reverse, 5'-TGAGCTAGTCCTTGA CTAACCT-3'; SIRT1 forward, 5'-TAGCCTTGTCAGATA AGGAAGGA-3' and reverse, 5'-ACAGCTTCACAGTCAACT TTGT-3'; proliferating cell nuclear antigen (PCNA) forward, 5'-TCA AGAAAATAAAACTAAGCTCT-3' and reverse, 5'-CTTCTAGGTTAACTAACCACA-3'; E-cadherin forward, 5'-TGTAACTTGCAATGGGCAGC-3' and reverse, 5'-CAA GCTCTCCTGCCATCTCC-3'; vimentin forward, 5'-ACG TCTTGACCTTGAACGCA-3' and reverse, 5'-TCTTGGCAG CCACACTTTCA-3'; U6 forward, 5'-CTCGCTTCGGCAGCA CA-3' and reverse, 5'-AACGCTTCACGAATTTGCGT-3'; and $\beta$-actin forward, 5'-CATGTACGTTGCTATCCAGGC-3' and reverse, 5'-CTCCTTAATGTCACGCACGAT-3'. Relative expression levels were quantified using the $2^{-\Delta \Delta C q}$ method (16).

Western blotting. Total protein was extracted from HBE, H1299 and A549 cells using RIPA lysis buffer (Beyotime Institute of Biotechnology). Protein concentration was determined using the BCA method (Beyotime Institute of Biotechnology). Protein samples $(15 \mu \mathrm{g} / \mathrm{lane})$ were separated via $10 \%$ SDS-PAGE, transferred onto PVDF membranes and subsequently blocked with $5 \%$ non-fat milk for $1 \mathrm{~h}$ at room temperature. The 
A

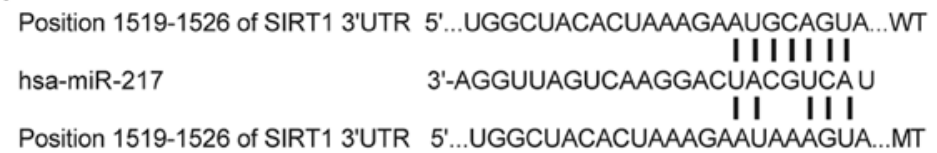

B

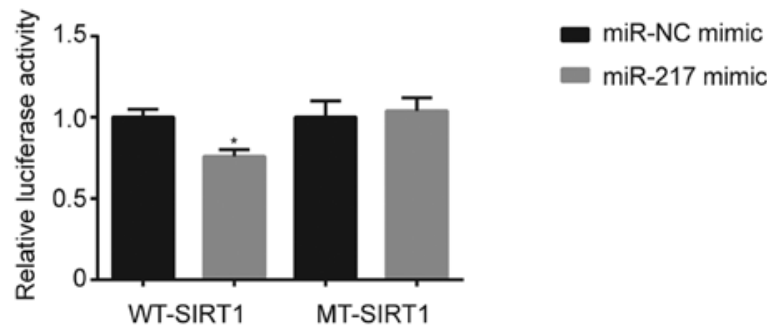

Figure 2. SIRT1 is the target gene of miR-217. (A) TargetScan analysis demonstrated that miR-217 was complementary to the 3'-UTR of SIRT1. (B) The WT plasmid was demonstrated to bind to miR-217, while the MT plasmid did not. " $\mathrm{P}<0.05$ vs. miR-NC mimic. SIRT1, sirtuin 1; miR, microRNA; UTR, untranslated region; WT, wild type; MT, mutant; NC, negative control.

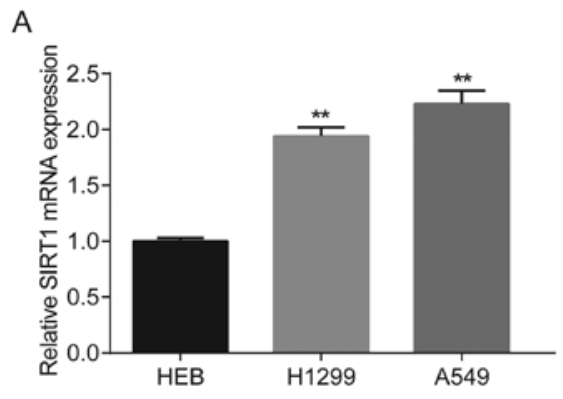

B

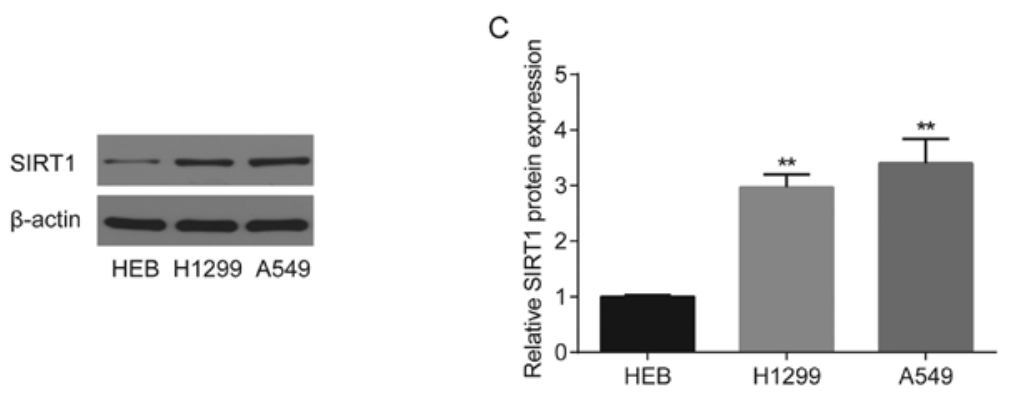

Figure 3. SIRT1 expression in the non-small cell lung cancer cell lines. SIRT1 (A) mRNA and (B and C) protein expression levels were significantly upregulated in H1299 and A549 cells compared with HBE cells. ${ }^{* *}$ P $<0.01$ vs. HEB. SIRT1, sirtuin 1.

membranes were incubated with primary antibodies against: SIRT1 (1:1,000; cat. no. 9475), PCNA (1:1,000; cat. no. 13110), E-cadherin $(1: 1,000$; cat. no. 3195), vimentin $(1: 1,000$; cat. no. 5741), p-AMPK $\alpha$ (1:1,000; cat. no. 2537), p-mTOR (1:1,000; cat. no. 5536), AMPK $\alpha$ (1:1,000; cat. no. 5832), mTOR (1:1,000; cat. no. 2983) and $\beta$-actin (1:2,000; cat. no. 4970) overnight at $4^{\circ} \mathrm{C}$ (all purchased from Cell Signaling Technology, Inc.). Following the primary incubation, membranes were incubated with anti-rabbit IgG, HRP-linked secondary antibodies (1:3,000; cat. no. 7074; Cell Signaling Technology, Inc.) for $2 \mathrm{~h}$ at room temperature. Protein bands were visualized using enhanced chemiluminescence (Amersham; Cytiva).

Statistical analysis. Statistical analysis was performed using SPSS 15.0 software (SPSS, Inc.). All experiments were performed in triplicate and data are presented as the mean \pm standard deviation. Student's t-test was used to compare differences between two groups, while one-way ANOVA and Tukey's post hoc test were used to compare differences between multiple groups. $\mathrm{P}<0.05$ was considered to indicate a statistically significant difference.

\section{Results}

miR-217 expression in the NSCLC cell lines. The results demonstrated that miR-217 expression was significantly downregulated in the NSCLC cells (H23, H292, H1299 and A549) compared with HBE cells $(\mathrm{P}<0.05$ and $\mathrm{P}<0.01$; Fig. 1$)$. Among the four NSCLC cell lines, H1299 and A549 cells had the lowest miR-217 expression levels; thus, these cell lines were selected for subsequent experimentation.

SIRT1 is the target gene of miR-217. TargetScan analysis demonstrated that miR-217 was complementary to the 3'-UTR of SIRT1 (Fig. 2A). The results of the dual-luciferase reporter assay further validated that co-transfection with miR-217 

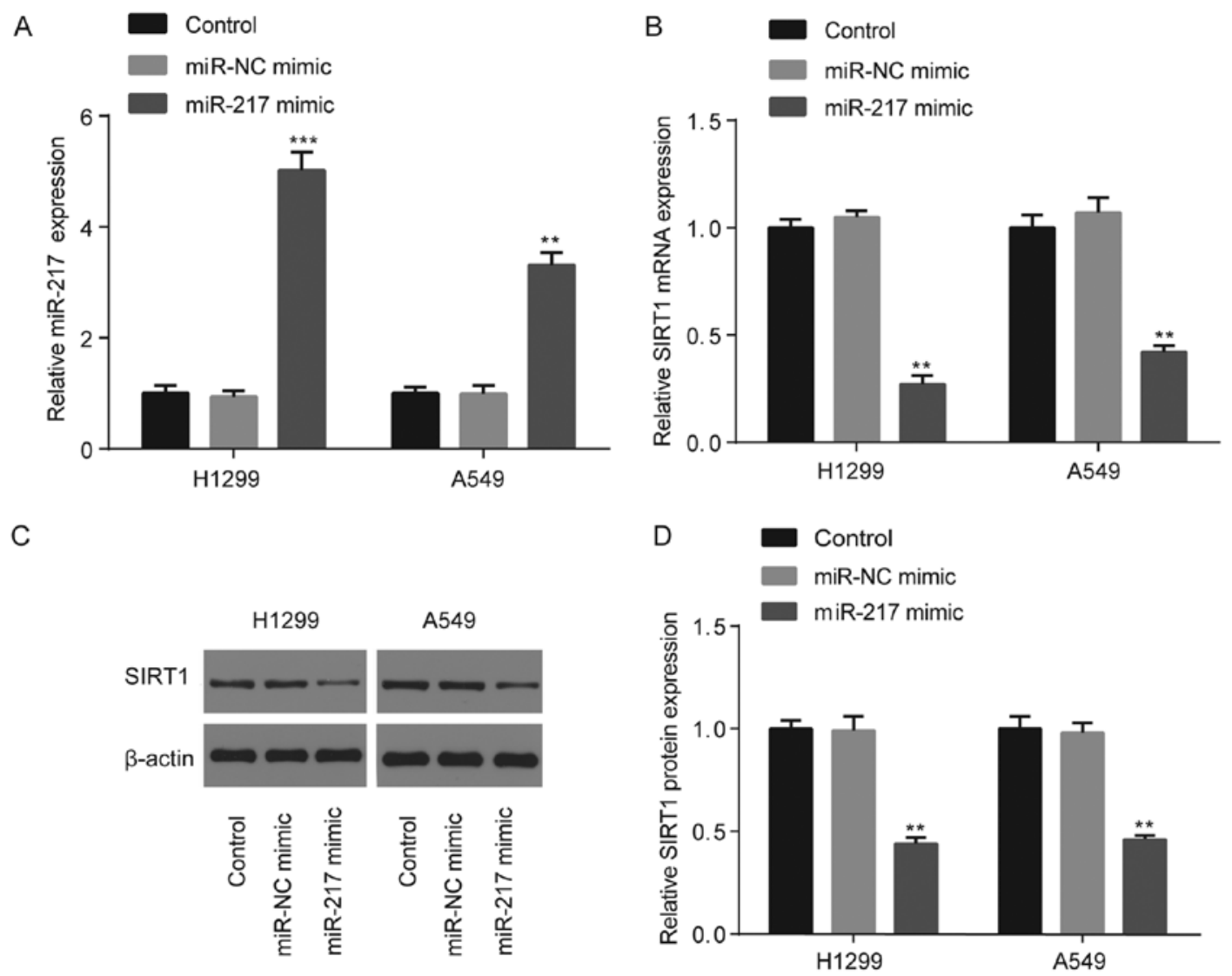

Figure 4. Transfection with miR-217 mimic in non-small cell lung cancer cells. (A) miR-217 expression was significantly upregulated in the miR-217 mimic group compared with the miR-NC mimic group. SIRT1 (B) mRNA and (C and D) protein expression levels were significantly downregulated in the miR-217 mimic group compared with the miR-NC mimic group. ${ }^{* *} \mathrm{P}<0.01,{ }^{* * * *} \mathrm{P}<0.001$ vs. miR-NC mimic. miR, microRNA; NC, negative control; SIRT1, sirtuin 1 .

mimic decreased the relative luciferase activity of cells transfected with WT-SIRT1 $(\mathrm{P}<0.05)$; however, no significant changes were observed following transfection with MT-SIRT1 (Fig. 2B).

SIRT1 expression in the NSCLC cell lines. The results demonstrated that both SIRT1 mRNA (Fig. 3A) and protein (Fig. 3B and C) expression levels were significantly upregulated in H1299 and A549 cells compared with HBE cells $(\mathrm{P}<0.01)$.

Transfection with miR-217 mimic in NSCLC cells. No significant differences in miR-217 expression were observed between the control (un-transfected group) and miR-NC mimic groups; however, miR-217 expression significantly increased following transfection with miR-217 mimic ( $\mathrm{P}<0.01$; Fig. 4A). In addition, no significant differences in SIRT1 mRNA and protein expression were observed between the control and miR-NC mimic groups; however, SIRT1 mRNA and protein expression significantly decreased following transfection with miR-217 mimic ( $\mathrm{P}<0.01$; Fig. 4B-D).

Transfection with pcDNA3.1-SIRT1 in NSCLC cells. No significant differences in SIRT1 mRNA or protein expression were observed between the control (un-transfected group) and pcDNA3.1 groups; however, SIRT1 mRNA and protein expression significantly increased following transfection with pcDNA3.1-SIRT1 ( $\mathrm{P}<0.01$ and $\mathrm{P}<0.001$; Fig. 5A-C).
SIRT1 reverses the miR-217 mimic-induced inhibition of NSCLC cell proliferation and invasion. To further determine whether the effects of miR-217 on NSCLC cell proliferation and invasion were mediated by SIRT1, miR-217 mimic and pcDNA3.1-SIRT1 were co-transfected into H1299 and A549 cells. The results demonstrated that miR-217 mimic significantly decreased $(\mathrm{P}<0.01)$ cell proliferation (Fig. 6A and $\mathrm{B})$, and PCNA mRNA (Fig. 6C) and protein expression levels of H1299 and A549 cells (Fig. 6D and E), the effects of which were partially rescued following overexpression of SIRT1 $(\mathrm{P}<0.05)$.

miR-217 mimic significantly decreased $(\mathrm{P}<0.01)$ cell invasion (Fig. 7A) and downregulated vimentin mRNA levels (Fig. 7B), while significantly upregulating E-cadherin mRNA levels (Fig. 7C) of H1299 and A549 cells. These effects were partially rescued following overexpression of SIRT1 $(\mathrm{P}<0.05)$. The protein profiles of vimentin and E-cadherin were similar to that of their mRNA levels (Fig. 7D-F).

SIRT1 reverses the miR-217 mimic-induced NSCLC cell apoptosis. To further determine whether the effects of miR-217 on NSCLC cell apoptosis were mediated by SIRT1, miR-217 mimic and pcDNA3.1-SIRT1 were co-transfected into H1299 and A549 cells. The results demonstrated that miR-217 mimic significantly increased $(\mathrm{P}<0.01)$ cell apoptosis in H1299 (Fig. 8A and B) and A549 (Fig. 8C and D) cells, the 

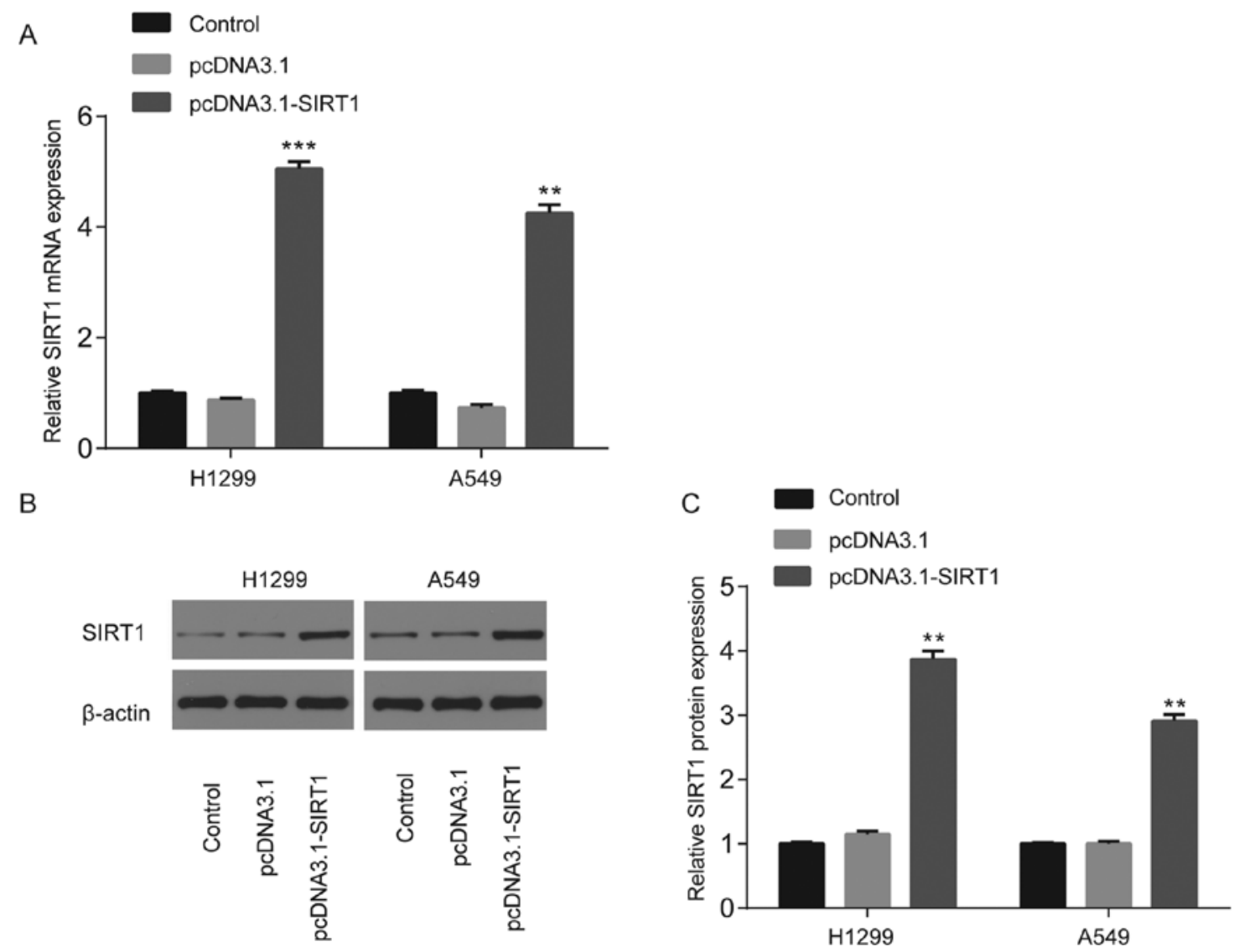

Figure 5. Transfection with pcDNA3.1-SIRT1 in non-small cell lung cancer cells. SIRT1 (A) mRNA and (B and C) protein expression significantly increased in the pcDNA3.1-SIRT1 group compared with the pcDNA3.1 group. ${ }^{* * *} \mathrm{P}<0.01,{ }^{* * *} \mathrm{P}<0.001$ vs. pcDNA3.1. SIRT1, sirtuin 1.

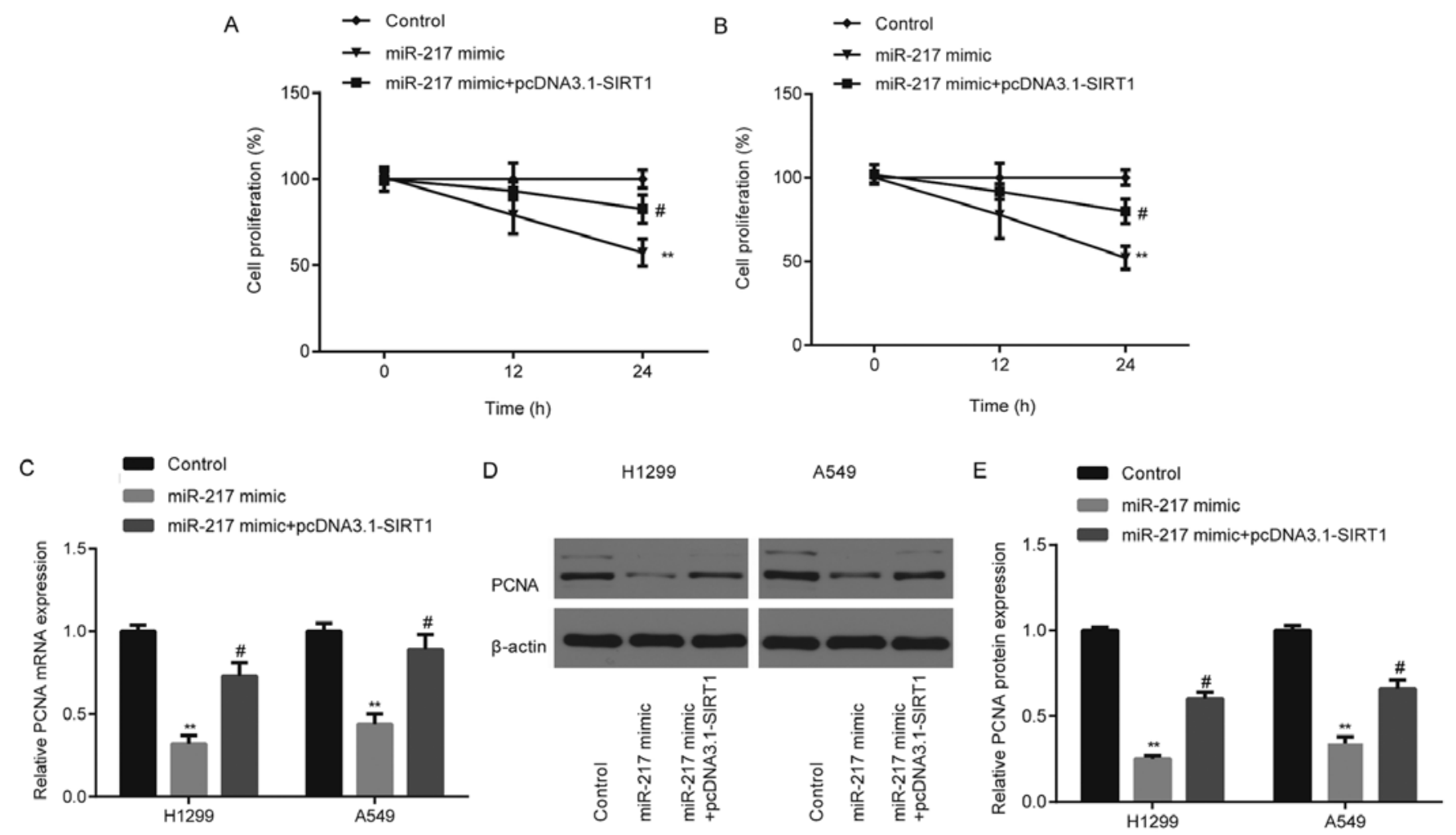

Figure 6. SIRT1 reverses the miR-217 mimic-induced inhibition of non-small cell lung cancer cell proliferation. (A and B) Transfection with miR-217 mimic significantly decreased cell proliferation, and (C) PCNA mRNA and (D and E) protein expression of H1299 and A549 cells, the effects of which were partially rescued following overexpression of SIRT1. ${ }^{* *} \mathrm{P}<0.01$ vs. control; ${ }^{*} \mathrm{P}<0.05$ vs. miR-217 mimic. SIRT1, sirtuin 1; miR, microRNA; PCNA, proliferating cell nuclear antigen. 

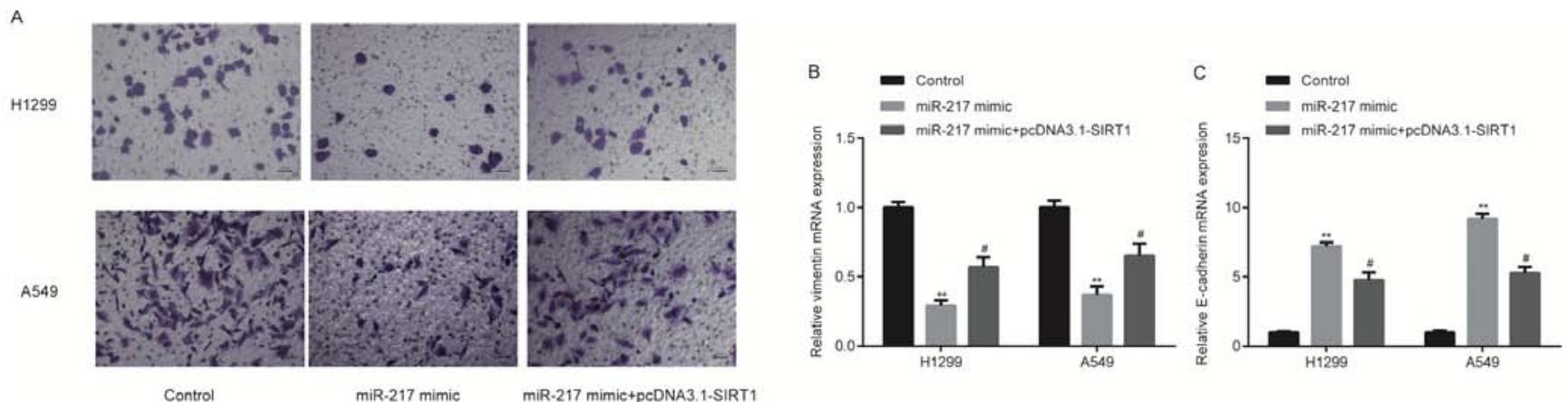

D
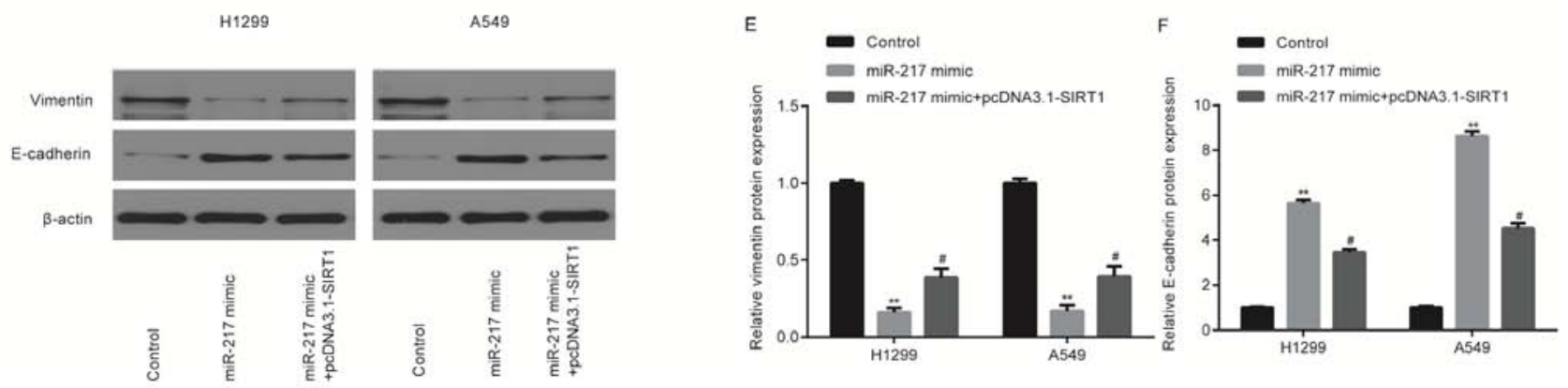

Figure 7. SIRT1 reverses the miR-217 mimic-induced inhibition of non-small cell lung cancer cell invasion. (A) Transfection with miR-217 mimic significantly decreased cell invasion (magnification, x100) and (B) vimentin mRNA expression, and (C) significantly increased E-cadherin mRNA expression of H1299 and A549 cells, the effects of which were partially rescued following overexpression of SIRT1. (D-F) The protein profiles of vimentin and E-cadherin were similar to that of their mRNA expression levels. ${ }^{* *} \mathrm{P}<0.01$ vs. control; ${ }^{*} \mathrm{P}<0.05$ vs. miR-217 mimic. SIRT1, sirtuin 1; miR, microRNA.

A

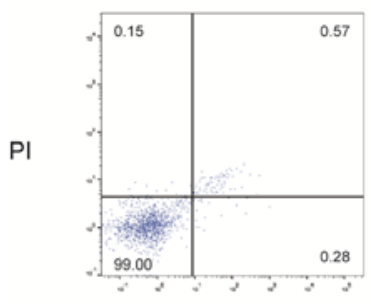

Control

C

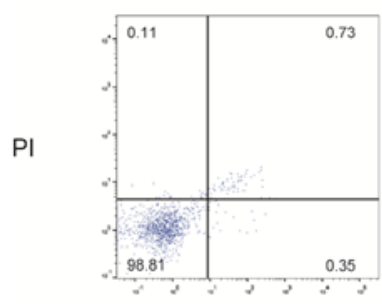

Control
H1299

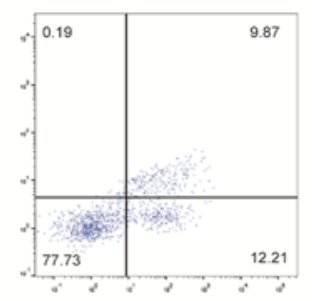

ANNEXIN-V

miR-217 mimic

A549

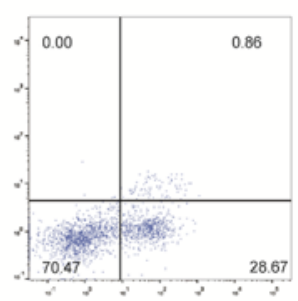

ANNEXIN-V

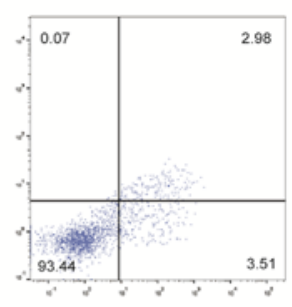

miR-217 mimic+pcDNA3.1-SIRT1

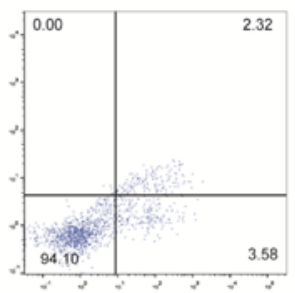

miR-217 mimic+pcDNA3.1-SIRT1

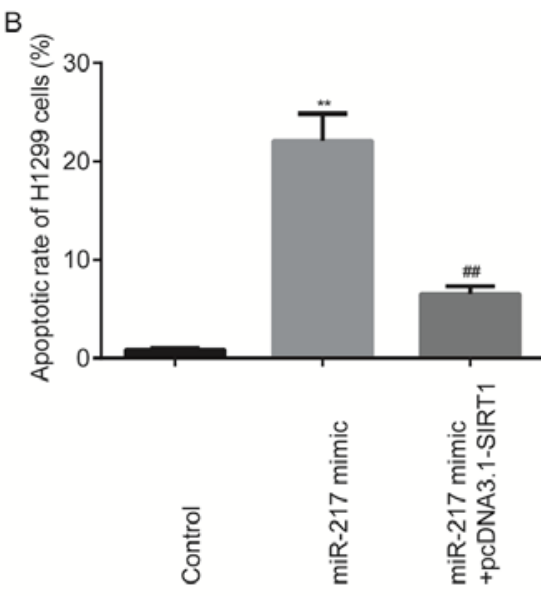

D

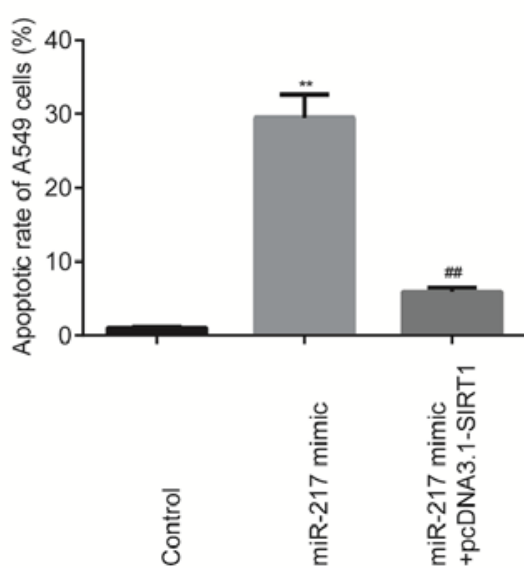

Figure 8. SIRT1 reverses the miR-217 mimic-induced apoptosis of non-small cell lung cancer cells. miR-217 mimic significantly increased apoptosis of (A and B) H1299 and (C and D) A549 cells, the effects of which were partially rescued following overexpression of SIRT1. ${ }^{* *} \mathrm{P}<0.01$ vs. control; ${ }^{\# \#} \mathrm{P}<0.001$ vs. miR-217 mimic. SIRT1, sirtuin 1; miR, microRNA; PI, propidium iodide. 
A
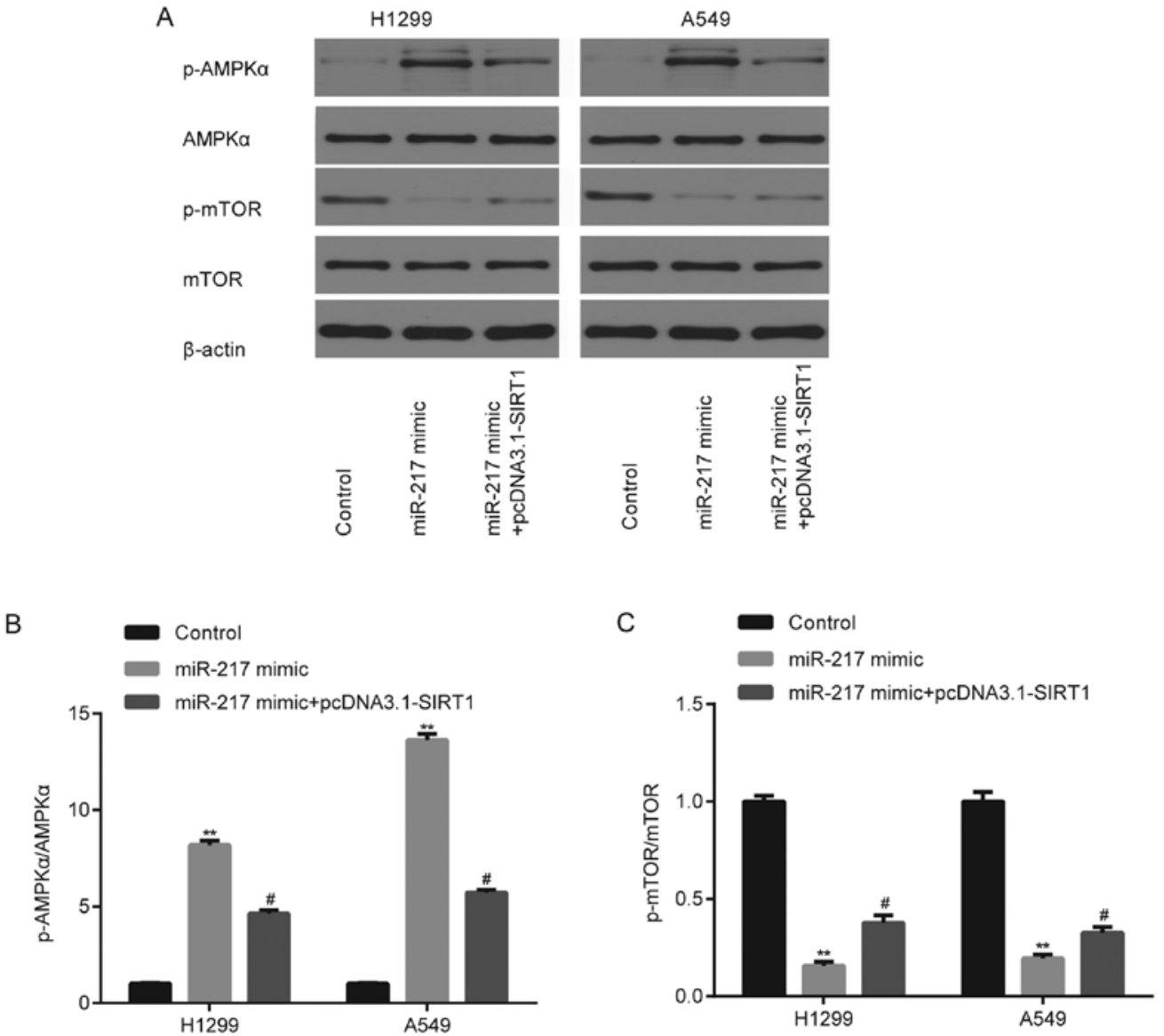

Figure 9. SIRT1 reverses the miR-217 mimic-induced decrease of p-AMPK $\alpha$ and increase of p-mTOR in non-small cell lung cancer cells. (A and B) miR-217 mimic significantly decreased p-AMPK $\alpha$ protein expression and (A and C) increased p-mTOR protein expression of H1299 and A549 cells, the effects of which were partially rescued following overexpression of SIRT1. ${ }^{* *} \mathrm{P}<0.01$ vs. control; ${ }^{*} \mathrm{P}<0.05$ vs. miR-217 mimic. SIRT1, sirtuin 1 ; miR, microRNA; $\mathrm{p}$-AMPK $\alpha$, phosphorylated amp-activated protein kinase $\alpha$.

effects of which were partially rescued following overexpression of SIRT1 $(\mathrm{P}<0.01)$.

SIRT1 reverses the miR-217 mimic-induced decrease of phosphorylated ( $p)-A M P K \alpha$ and increase of $p-m T O R$ in NSCLC cells. To further determine whether the effects of miR-217 and SIRT1 on NSCLC progression involve the p-AMPK $\alpha$ and p-mTOR signaling pathways, miR-217 mimic and pcDNA3.1-SIRT1 were co-transfected into H1299 and A549 cells. The results demonstrated that miR-217 mimic significantly downregulated $(\mathrm{P}<0.01) \mathrm{p}-\mathrm{AMPK} \alpha$ protein expression (Fig. 9A and B) and upregulated p-mTOR protein expression (Fig. 9A and C) in H1299 and A549 cells. These effects were partially rescued following overexpression of SIRT1 $(\mathrm{P}<0.05)$.

\section{Discussion}

miR-217 has been reported to act as a tumor suppressor in several types of diseases. For example, miR-217 represses HeLa cell migration and invasion through MAPK1 in cervical cancer (17); it inhibits M2-like macrophage polarization by decreasing IL-6 in ovarian cancer (18); and it inhibits the proliferation and promotes apoptosis in hepatocytes by targeting NAT2 in liver injury (19). In addition, miR-217 expression is downregulated in lung cancer, while overexpression of miR-217 has been demonstrated to inhibit the proliferation, migration and invasion, and induce apoptosis of the lung cancer cell lines, SPC-A-1 and A549 by targeting KRAS (10). miR-217 expression is also downregulated by CSE in $\mathrm{HBE}$ cells, while miR-217 regulation of EZH2/H3K27me3 by MALAT1 is involved in CSE-induced malignant transformation of HBE cells (11). Furthermore, circ_0023404 promotes cell proliferation, migration and invasion of the NSCLC cell lines, A549 and H1299 by targeting the miR-217/ZEB1 axis (20). The inhibitory effects of miR-217 overexpression on proliferation and invasion have been previously reported, along with the promotional effects of miR-217 overexpression on the apoptosis of the NSCLC cell lines, H1299 and A549 (10,20). The present study aimed to investigate other potential mRNA targets of miR-217 in NSCLC.

Increasing evidence has indicated that SIRT1 serves as an oncogene. For example, SIRT1 inactivates p53, thus inhibiting the functions of several tumor suppressors (21), and SIRT1 has also been demonstrated to regulate multiple tumor suppressors epigenetically $(22,23)$. Furthermore, SIRT1 was identified to be overexpressed in mouse and human prostate cancer (24), while inhibition of SIRT1 has been reported to repress the progression of several types of tumors, including colon, prostate and melanoma cancer (25). SIRT1 is associated with the poor prognosis of patients with NSCLC (13), 
and is overexpressed in metastatic brain tissues of patients with NSCLC, where it promotes migration of A549 cells (14). The results of the present study confirmed that SIRT1 is a target of miR-217, and serves as an oncogene in NSCLC. Overexpression of SIRT1 reversed the inhibitory effects of miR-217 mimic on cell proliferation and migration, and the promotional effects of miR-217 mimic on cell apoptosis in the NCSLC cell lines, H1299 and A549. Notably, during the process of the present study, a newly published study demonstrated the involvement of miR-217 and SIRT1 in lung cancer, suggesting that miR-217 inhibits the proliferation, migration, and invasion of the human pulmonary adenocarcinoma brain metastasis cell line PC-14/B, by targeting SIRT1 and activating the P53/KAI1 signaling pathway (26). Other studies reported that downregulation of miR-217 attenuates atherosclerosis by inhibiting macrophage apoptosis and inflammation via SIRT1 (27), miR-217 decreases proliferation, migration, and invasion of osteosarcoma by targeting SIRT1 (15), and downregulation of miR-217 mitigates inflammation and myocardial injury in children with viral myocarditis by regulating the $\mathrm{AMPK} / \mathrm{NF}-\kappa \mathrm{B}$ signaling pathway via SIRT1 (28). Consistent with previous findings, the results of the present study verified the interaction between miR-217 and SIRT1 in the proliferation, invasion and apoptosis of NSCLC cells. However, the downstream molecules responsible for the aforementioned effects of SIRT1 are yet to be elucidated.

As a downstream effector of SIRT1, AMPK functions as a metabolic stress sensor in HepG2 cells (29). In addition to AMPK-mediated lysosome biogenesis in the tumor growth of lung cancer (30), AMPK is indispensable for the Ginsenoside metabolite compound $\mathrm{K}$ induced apoptosis of NSCLC cells (31), as well as downregulation of TUFM induced invasion of NSCLC cells (32). mTOR is an oncogene that mediates cell survival (33) and is indispensable for apoptosis (34), and the MetaLnc9 induced metastasis of NSCLC (35). Activation of AMPK negatively regulates mTOR expression (36). Notably, this association has been previously observed in NSCLC $(31,37)$, suggesting the negative regulation of mTOR via the LKB1-AMPK signaling pathway. Taken together, the results of the present study suggest that miR-217 may inhibit AMPK phosphorylation and promote mTOR phosphorylation by targeting SIRT1. However, further studies are required to assess other key factors involved in mTOR signalization. Also, the present study failed to confirm the function of miR-217 in the in vivo experiments, which will be investigated in the future.

In conclusion, the results of the present study demonstrated that miR-217 inhibited NSCLC cell proliferation and invasion, and induced NCLC cell apoptosis by targeting SIRT1 and inactivating the SIRT1-mediated p-AMPK/p-mTOR signaling pathway.

\section{Acknowledgements}

Not applicable.

\section{Funding}

No funding was received.

\section{Availability of data and materials}

The datasets used and/or analyzed during the current study are available from the corresponding author on reasonable request.

\section{Author's contributions}

GL and SZ conceived the present study, performed the experiments, and analyzed the data. SZ supervised and drafted the initial manuscript. Both authors read and approved the final manuscript.

\section{Ethics approval and consent to participate}

Not applicable.

\section{Patient consent for publication}

Not applicable.

\section{Competing interests}

The authors declare that they have no competing interests.

\section{References}

1. Brahmer JR, Govindan R, Anders RA, Antonia SJ, Sagorsky S, Davies MJ, Dubinett SM, Ferris A, Gandhi L, Garon EB, et al: The Society for Immunotherapy of Cancer consensus statement on immunotherapy for the treatment of nonsmall cell lung cancer (NSCLC). J Immunother Cancer 6: 75, 2018

2. Zhang L, Shi SB, Zhu Y, Qian TT and Wang HL: Long non-coding RNA ASAP1-IT1 promotes cell proliferation, invasion and metastasis through the PTEN/AKT signaling axis in non-small cell lung cancer. Eur Rev Med Pharmacol Sci 22: 142-149, 2018.

3. Hirsch FR, Scagliotti GV, Mulshine JL, Kwon R, Curran WJ Jr, Wu YL and Paz-Ares L: Lung cancer: Current therapies and new targeted treatments. Lancet 389: 299-311, 2017.

4. NSCLC Meta-analyses Collaborative Group; Arriagada R, Auperin A, Burdett S, Higgins JP, Johnson DH, Le Chevalier T, Le Pechoux C, Parmar MK, Pignon JP, et al: Adjuvant chemotherapy, with or without postoperative radiotherapy, in operable non-small-cell lung cancer: Two meta-analyses of individual patient data. Lancet 375: 1267-1277, 2010

5. Zeng H, Zheng R, Guo Y, Zhang S, Zou X, Wang N, Zhang L, Tang J, Chen J, Wei K, et al: Cancer survival in China, 2003-2005: A population-based study. Int J Cancer 136: 1921-1930, 2015.

6. Wu S, Zhao X, Wu S, Du R, Zhu Q, Fang H, Zhang X, Zhang C, Zheng W, Yang J and Feng H: Overexpression of B 7-H3 correlates with aggressive clinicopathological characteristics in non-small cell lung cancer. Oncotarget 7: 81750-81756, 2016.

7. Bartel DP: MicroRNAs: Genomics, biogenesis, mechanism, and function. Cell 116: 281-297, 2004.

8. Liu T, Wu X, Chen T, Luo Z and Hu X: Downregulation of DNMT3A by miR-708-5p inhibits lung cancer stem cell-like phenotypes through repressing Wnt $/ \beta$-catenin signaling. Clin Cancer Res 24: 1748-1760, 2018.

9. Paliouras AR, Monteverde T and Garofalo M: Oncogene-induced regulation of microRNA expression: Implications for cancer initiation, progression and therapy. Cancer Lett 421: 152-160, 2018.

10. Guo J, Feng Z, Huang Z, Wang H and Lu W: MicroRNA-217 functions as a tumour suppressor gene and correlates with cell resistance to cisplatin in lung cancer. Mol Cells 37: 664-671, 2014.

11. LuL,LuoF,Liu Y,Liu X, Shi L,LuXandLiu Q: Posttranscriptional silencing of the lncRNA MALAT1 by miR-217 inhibits the epithelial-mesenchymal transition via enhancer of zeste homolog 2 in the malignant transformation of HBE cells induced by cigarette smoke extract. Toxicol Appl Pharmacol 289: 276-285, 2015. 
12. Wang Y, Bi Y, Chen X, Li C, Li Y, Zhang Z, Wang J, Lu Y, Yu Q, $\mathrm{Su} \mathrm{H}$, et al: Histone deacetylase SIRT1 negatively regulates the differentiation ofinterleukin-9-producing $\mathrm{CD}^{+}{ }^{+} \mathrm{T}$ cells. Immunity 44: 1337-1349, 2016.

13. Grbesa I, Pajares MJ, Martínez-Terroba E, Agorreta J, Mikecin AM, Larráyoz M, Idoate MA, Gall-Troselj K, Pio R and Montuenga LM: Pression of sirtuin 1 and 2 is associated with poor prognosis in non-small cell lung cancer patients. PLoS One 10: e0124670, 2015.

14. Han L, Liang XH, Chen LX, Bao SM and Yan ZQ: SIRT1 is highly expressed in brain metastasis tissues of non-small cell lung cancer (NSCLC) and in positive regulation of NSCLC cell migration. Int J Clin Exp Pathol 6: 2357-2365, 2013.

15. He S, Wang Z, Tang H, Dong J, Qu Y and Lv J: miR-217 inhibits proliferation, migration, and invasion by targeting SIRT1 in osteosarcoma. Cancer Biother Radiopharm 34: 264-270, 2019.

16. Livak KJ and Schmittgen TD: Analysis of relative gene expression data using real-time quantitative PCR and the 2(-Delta Delta C(T)) method. Methods 25: 402-408, 2001

17. Zhu L, Yang S and Wang J: miR-217 inhibits the migration and invasion of HeLa cells through modulating MAPK1. Int J Mol Med 44: 1824-1832, 2019.

18. Jiang B, Zhu SJ, Xiao SS and Xue M: miR-217 inhibits M2-Like macrophage polarization by suppressing secretion of interleukin-6 in ovarian cancer. Inflammation 42: 1517-1529, 2019.

19. Yang CL,Zheng XL, Ye K, and Xue M: Effects of microRNA-217 on proliferation, apoptosis, and autophagy of hepatocytes in rat models of CCL4-induced liver injury by targeting NAT2. J Cell Physiol 234: 3410-3424, 2019.

20. Liu C, Zhang Z and Qi D: Circular RNA hsa_circ_0023404 promotes proliferation, migration and invasion in non-small cell lung cancer by regulating miR-217/ZEB1 axis. Onco Targets Ther 12: 6181-6189, 2019.

21. Vaziri H, Dessain SK, Ng Eaton E, Imai SI, Frye RA, Pandita TK Guarente L and Weinberg RA: hSIR2(SIRT1) functions as an NAD-dependent p53 deacetylase. Cell 107: 149-159, 2001.

22. Wong S and Weber JD: Deacetylation of the retinoblastoma tumour suppressor protein by SIRT1. Biochem J 407: 451-460, 2007.

23. Wang C, Chen L, Hou X, Li Z, Kabra N, Ma Y, Nemoto S, Finkel T, Gu W, Cress WD and Chen J: Interactions between E2F1 and SirT1 regulate apoptotic response to DNA damage. Nat Cell Biol 8: 1025-1031,2006.

24. Huffman DM, Grizzle WE, Bamman MM, Kim JS, Eltoum IA, Elgavish A and Nagy TR: SIRT1 is significantly elevated in mouse and human prostate cancer. Cancer Res 67: 6612-6618, 2007.

25. Alves-Fernandes DK and Jasiulionis MG: The role of SIRT1 on DNA damage response and epigenetic alterations in cancer. Int J Mol Sci 20: 3153, 2019

26. Jiang W, Hou L, Wei J, Du Y, Zhao Y, Deng X and Lin X: Hsa-miR-217 inhibits the proliferation, migration, and invasion in non-small cell lung cancer cells via targeting SIRT1 and P53/KAI1 signaling. Balkan Med J 37: 208-214, 2020.
27. Zhang L, Chen J,He Q, Chao Z,Li X and Chen M: MicroRNA-217 is involved in the progression of atherosclerosis through regulating inflammatory responses by targeting sirtuin 1 . Mol Med Rep 20: 3182-3190, 2019.

28. Xia K, Zhang Y and Sun D: miR-217 and miR-543 downregulation mitigates inflammatory response and myocardial injury in children with viral myocarditis by regulating the SIRT1/AMPK/NF- $\kappa$ B signaling pathway. Int J Mol Med 45: 634-646, 2020

29. Salomone F, Barbagallo I, Godos J, Lembo V, Currenti W, Cinà D, Avola R, D'Orazio N, Morisco F, Galvano F and Li Volti G: Silibinin restores NAD (+) levels and induces the SIRT1/AMPK pathway in non-alcoholic fatty liver. Nutrients 9: 10, 2017.

30. Patra KC, Weerasekara VK and Bardeesy N: AMPK-mediated lysosome biogenesis in lung cancer growth. Cell Metab 29: 238-240, 2019 .

31. Li C, Dong Y, Wang L, Xu G, Yang Q, Tang X, Qiao Y and Cong Z Ginsenoside metabolite compound $\mathrm{K}$ induces apoptosis and autophagy in non-small cell lung cancer cells via AMPK-mTOR and JNK pathways. Biochem Cell Biol 97: 406-414, 2019.

32. He K, Guo X, Liu Y, Li J, Hu Y, Wang D and Song J: TUFM downregulation induces epithelial-mesenchymal transition and invasion in lung cancer cells via a mechanism involving AMPK-GSK3 $\beta$ signaling. Cell Mol Life Sci 73: 2105-2121, 2016.

33. Yan G, Ru Y, Wu K, Yan F, Wang Q, Wang J, Pan T, Zhang M, Han H, Li X and Zou L: GOLM1 promotes prostate cancer progression through activating PI3K-AKT-mTOR signaling. Prostate 78: 166-177, 2017.

34. Liu G, Pei F, Yang F, Li L, Amin AD, Liu S, Buchan JR and Cho WC: Role of autophagy and apoptosis in non-small-cell lung cancer. Int J Mol Sci 18: 367, 2017.

35. Yu T, Zhao Y, Hu Z, Li J, Chu D, Zhang J, Li Z, Chen B, Zhang X, Pan H, et al: MetaLnc9 facilitates lung cancer metastasis via a PGK1-activated AKT/mTOR pathway. Cancer Res 77: 5782-5794, 2017.

36. Zhao W, Peng F, Shu M, Liu H, Hou X, Wang X, Ye J, Zhao B, Wang $\mathrm{K}$, Zhong $\mathrm{C}$, et al: Isogambogenic acid inhibits the growth of glioma through activation of the AMPK-mTOR pathway. Cell Physiol Biochem 44: 1381-1395, 2017.

37. Dong LX, Sun LL, Zhang X, Pan L, Lian LJ, Chen Z and Zhong DS: Negative regulation of mTOR activity by LKB1-AMPK signaling in non-small cell lung cancer cells. Acta Pharmacol Sin 34: 314-318, 2013.

c) (i) $($ This work is licensed under a Creative Commons Attribution-NonCommercial-NoDerivatives 4.0 International (CC BY-NC-ND 4.0) License. 